\title{
Principles and characteristics of a new generation plasma immersion ion implanter
}

\author{
P. K. Chu a) and B. Y. Tang \\ Department of Physics and Materials Science, City University of Hong Kong, Hong Kong \\ Y. C. Cheng \\ University of Hong Kong, Hong Kong \\ P. K. Ko \\ School of Engineering, Hong Kong University of Science and Technology, Hong Kong \\ (Received 25 November 1996; accepted for publication 5 January 1997)
}

\begin{abstract}
A new generation multipurpose plasma immersion ion implanter (PIII) was custom designed, constructed, and installed in the City University of Hong Kong. The system is designed for general R\&D applications in metallurgy, tribology, surface modification, and fabrication of novel materials. Using the new rf ion source in conjunction with the internal antenna system, the plasma density achieves excellent uniformity both laterally and axially. The system also incorporates two metal sources, including four metal arc sources and a sputtering electrode, so that multiple metal deposition and implantation steps can be performed in succession in the same equipment without exposing the samples to air. This capability can be critical to the study of surface properties and corrosion resistance. This article describes the design objectives, the novel features, and the characteristics of this new generation PIII equipment. (C) 1997 American Institute of Physics. [S0034-6748(97)04604-2]
\end{abstract}

\section{INTRODUCTION}

Conventional ion beam ion implantation (IBII) is a versatile tool for materials modification and semiconductor processing. It has been shown to be effective in enhancing the mechanical properties such as surface hardness, wear resistance, and corrosion resistance of materials, as well as modifying their electrical and optical properties. ${ }^{1-5}$ As a line-ofsight process, however, IBII has some shortcomings when complex-shaped targets must be implanted. Techniques such as raster scanning, sample rotation, and target masking are required for uniform implantation. In addition to the added technical complexities, a higher cost results, and IBII has hitherto not received widespread acceptance in the nonsemiconductor industry.

Plasma immersion ion implantation is a novel surface modification technique that was originally conceived and developed by Conrad and co-workers at the University of Wisconsin. ${ }^{6-13}$ Initially called plasma source ion implantation (PSII), this method is now frequently referred to as plasma immersion ion implantation (PIII) in order to differentiate it from conventional beamline ion implantation encompassing plasma ion sources. In PIII, the specimens are surrounded by a plasma and pulse biased to a high negative potential relative to the chamber wall. Ions generated in the plasma shroud are accelerated across the sheath formed around the samples and implanted normally into the surface of the targets. Hence PIII circumvents the line-of-sight restriction inherent to IBII, eliminates complex focusing elements, and is thus potentially more economical. Moreover, being a nonline-of-sight process, large and complex-shaped

a)Electronic mail: appkchu@ @ityu.edu.hk specimens and multiple samples can be implanted simultaneously.

In spite of the tremendous potential offered by PIII, commercial instrumentation has not caught up and most PIII equipment tends to be built from existing hardware or cater to specific R\&D applications. A few years ago, we embarked on the design of a new generation PIII prototype that incorporates most of the features people want and addresses the needs of researchers in a myriad of areas, including tribology, metallurgy, semiconductor, and so on.$^{14}$ The prototype is intended to become the platform for future commercial multipurpose PIII equipment. In this article, we will discuss our design principles and describe the operation characteristics of the prototype.

\section{DESIGN PRINCIPLES}

Versatility being the most important issue, the equipment is designed to meet the following requirements.

(1) A myriad of experiments, including surface coating, ion plating, sputter deposition, recoil implantation, dynamic recoil implantation, ion beam mixing, bombardmentdiffusion coating, overlap implantation, and more, can be conducted in the same equipment either simultaneously or in succession without having to remove the samples from vacuum.

(2) A broad spectrum of ion sources, gas or solid, is available to implant practically all elements of interest.

(3) The sample chamber is big enough to handle common samples and incorporates built-in safety features.

(4) Various means to ignite and sustain the plasma are provided to cater to different applications.

(5) Diagnostics tools are built in.

(6) Most instrument controls are via computer control. 


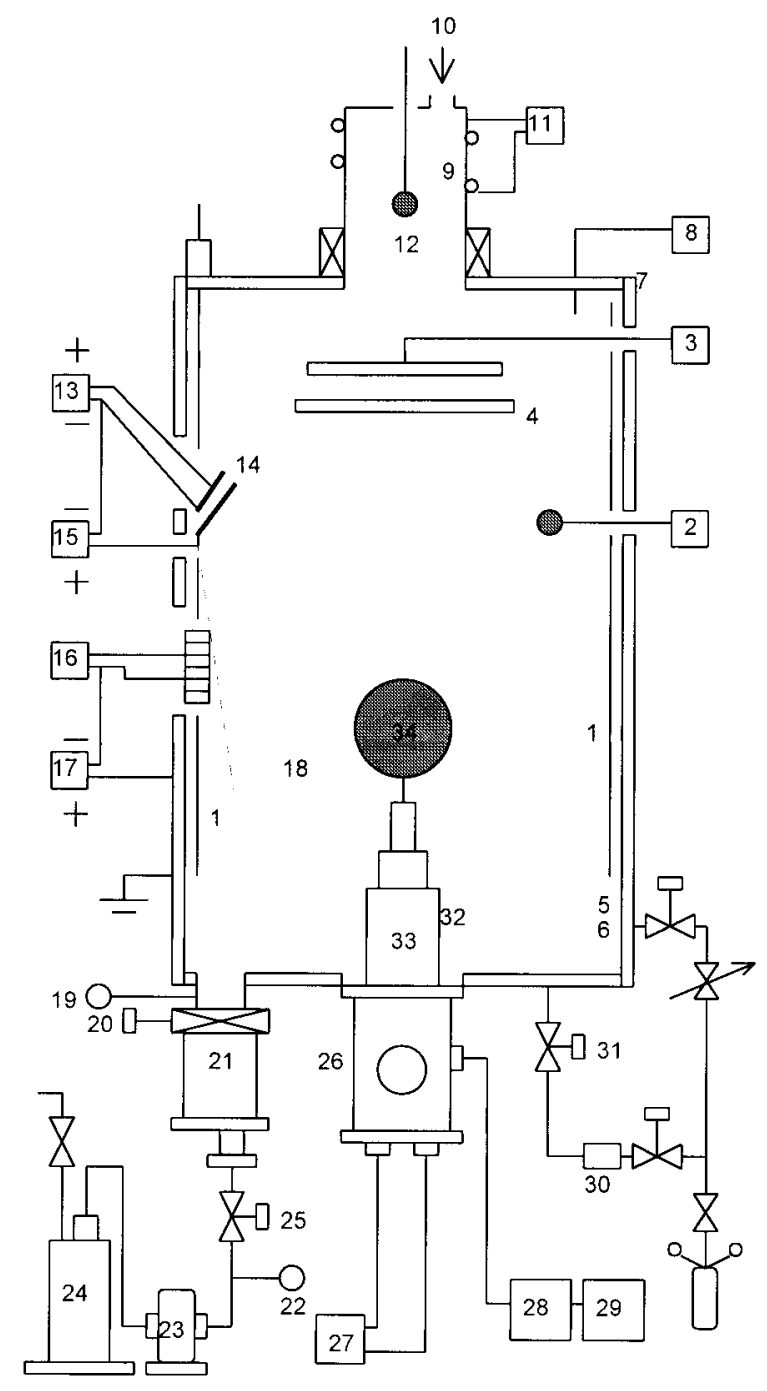

FIG. 1. Schematic of the City University's PIII equipment. (1) rf antenna system; (2) radial Langmuir probe system; (3) sputtering electrode system; (4) shutter; (5) liners; (6) chamber wall; (7) permanent magnets; (8) temperature measurement module; (9) rf plasma chamber; (10) gas inlet; (11) rf generator; (12) axial Langmuir probe system; (13) trigger power supply; (14) MEVVA plasma sources; (15) arc power supply for MEVVA plasma sources; (16) filament power supply; (17) discharge bias power supply; (18) vacuum chamber; (19) ionization gauge; (20) gate valve; (21) turbomolecular pump; (22) CT; (23) Roots pump; (24) mechanical pump; (25) electromagnetic valve; (26) oil barrel and HV feedthrough; (27) oil cooling system; (28) high voltage pulse modulator; (29) dc high voltage power supply; (30) mass flowmeter; (31) gas feeding system; (32) Pyrex shielding cylinder; (33) HV target stage; (34) workpiece.

\section{CHARACTERISTICS AND OPERATING PRINCIPLES}

A schematic of the PIII equipment is exhibited in Fig. 1 and a picture of it is displayed in Fig. 2.

\section{A. Main vacuum chamber}

Considering the need to process complex-shaped and large samples, the sample chamber must be sufficiently big. Our equipment is designed to handle four spherical targets 6 $\mathrm{cm}$ in diameter simultaneously. Since the implantation region should be a cylinder $84 \mathrm{~cm}$ in diameter, a cylindrical stainless steel chamber $100 \mathrm{~cm}$ in diameter is required. The

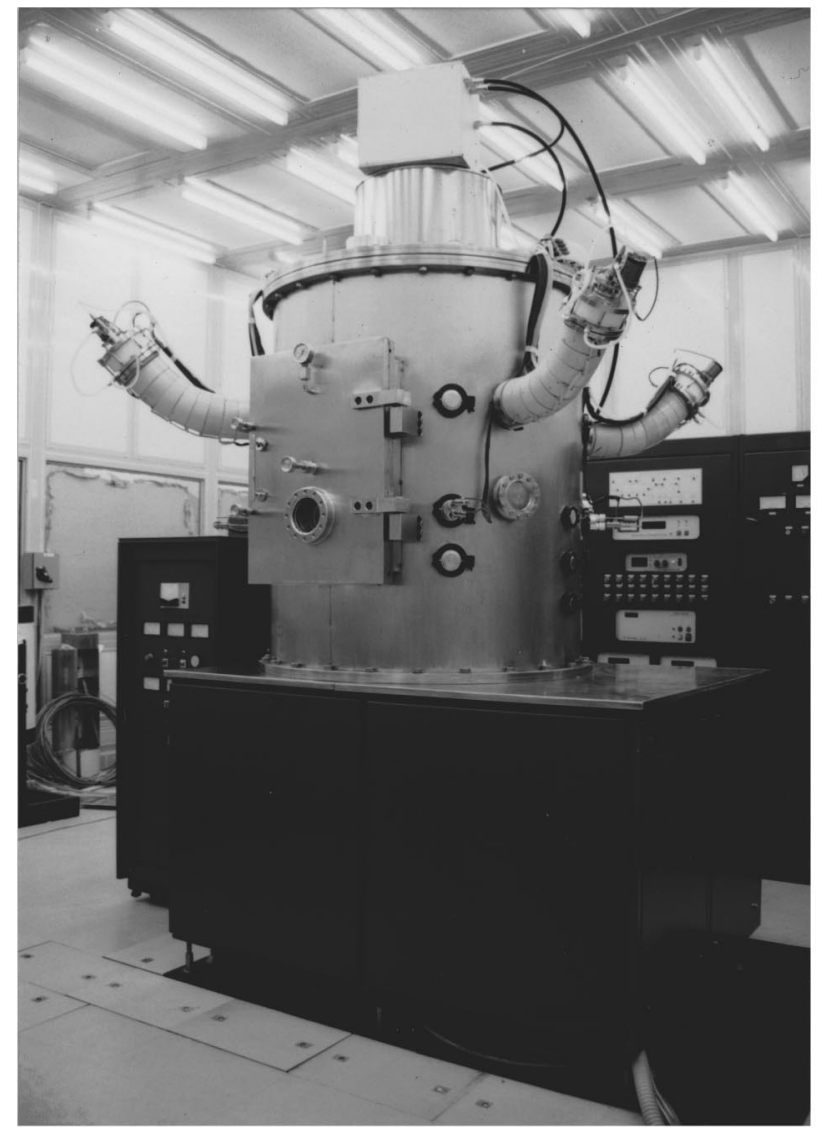

FIG. 2. Picture of the City University's PIII equipment.

axial height of the chamber must be large enough to accommodate the high voltage target stage, high voltage support, module under the top flange, and the targets. It is chosen to be $120 \mathrm{~cm}$ and the chamber volume is $940 \ell$. The vacuum chamber is made of $\mathrm{Cr} 18 \mathrm{Ni}$ TTi stainless steel.

The thickness of the chamber wall is calculated by the following formula: ${ }^{15}$

$$
S_{0}=1.25 D(P L / E D)^{0.4},
$$

where $S_{0}$ is the thickness of the chamber wall in mm, $D$ is the inside diameter of the chamber in $\mathrm{mm}, P$ is atmospheric pressure in $\mathrm{kg} / \mathrm{cm}^{2}, L$ is the length of the chamber in $\mathrm{mm}$, and $E$ is the modulus of elasticity of the material at a maximum working temperature of $200{ }^{\circ} \mathrm{C} . S_{0}$ is calculated to be $4.23 \mathrm{~mm}$. The actual thickness of the chamber, which is equal to $S_{0}+C$, where $C$ is the additive quantity considering the surface status of the chamber wall, should be $6 \mathrm{~mm}$.

When the diameter of the cylindrical vacuum chamber is less than $150 \mathrm{~cm}$, planar flanges can be installed at both ends of the chamber. The flange thickness can be computed using the following relationship: ${ }^{15}$

$$
S=D[K P /(\sigma)]^{1 / 2}+C,
$$

where $S$ is the thickness of the planar flange in mm, $K$ is the structure coefficient $(K=0.4), P$ is atmospheric pressure in $\mathrm{kg} / \mathrm{cm}^{2},(\sigma)=1333 \mathrm{~kg} / \mathrm{cm}^{2}$, and $C$ is equal to $2.3 \mathrm{~mm}$. The calculated thickness of the flange is thus $19.6 \mathrm{~mm}$. 
There are 44 orifices on the sidewall as well as on the top and bottom flanges of the vacuum chamber. Their locations are (number of openings in parentheses):

(a) top flange: rf plasma source (1), metal vapor vacuum arc (MEVVA) plasma sources/plasma diagnosis (4), and multifilament modules and feedthroughs for the rf antennae (4);

(b) bottom flange: high voltage target stage (1), vacuum pumping system (1), future cryogenic pump (1), prepumping line (1), and gas feeding line (1);

(c) sidewall: MEVVA plasma sources and plasma diagnostic tools (8), multifilament modules (12), sputtering electrode and shutter (3), radial Langmuir probe (4), and rf antenna system feedthrough (3).

The MEVVA plasma sources, multifilament modules, sputtering electrode, shutter, and Langmuir probe can be mounted on different openings to accommodate odd-shape workpieces and other diagnostic tools.

The rectangular door of the chamber measures $500 \mathrm{~mm}$ $\times 700 \mathrm{~mm}$. It is mounted on the side of the chamber and provides access for sample loading, installation of auxiliary equipment, and so on. The door is composed of a door plate and rotation hinges operating on a compression mechanism. There is a vacuum pressure meter on the door to display the vacuum status (vacuum or atmospheric pressure). There is an internal shutter on the door to protect the viewing window from unintentional contamination and coating. The observation window is made of lead glass and measures $15 \mathrm{~cm}$ in diameter and $2 \mathrm{~cm}$ in thickness. It is mounted in the middle of the door.

Permanent magnets are installed on the outer surface of the main vacuum chamber to form a full cusp magnetic field to confine the electrons, reduce plasma loss to the chamber walls, and improve the plasma density uniformity. As $\mathrm{Sm}-\mathrm{Co}$ magnets are quite expensive, $\mathrm{Nd}-\mathrm{Fe}-\mathrm{B}$ magnets are employed. Their dimensions are $30 \mathrm{~mm} \times 10 \mathrm{~mm} \times 5 \mathrm{~mm}$. The maximum working temperature is $120{ }^{\circ} \mathrm{C}$ and cooling water lines are installed to prevent overheating of the magnets during operation.

The inside chamber walls are prone to contaminant deposition from the plasma and regular cleaning is necessary. Removable aluminum liners are installed inside the main vacuum chamber to facilitate cleaning and remounting.

Soft $\mathrm{x}$ rays are produced in high energy ion implantation as stray electrons can strike the chamber walls and surfaces and emit $x$ rays. In order to prevent these damaging photons from reaching the operators, the chamber is equipped with lead shields. Based on an accelerating voltage of $100 \mathrm{kV}$ and an average ion current of $50 \mathrm{~mA}$, it is calculated and experimentally proven that two layers of $3 \mathrm{~mm}$ lead sheets are adequate in blocking the $\mathrm{x}$ rays. The lead sheets are built into the chamber wall. There are also lead pockets covering the various windows and, as a result, the soft x-ray dose outside the instrument is much less than the acceptable $500 \mathrm{mrem} /$ year level.

\section{B. rf plasma source and antenna system}

There are a number of plasma sources commonly used in PIII systems. ${ }^{16}$ Each has advantages and drawbacks. For instance, an electron cyclotron resonance (ECR) source can deliver a high plasma density but is relatively expensive, and the high density is not needed in most applications. Therefore, a more economical rf design is chosen for our equipment. In a rf capacitively coupled glow discharge system, the plasma density and substrate bias are closely related. A high plasma density can be achieved with a high rf input power, but a high substrate bias and consequently sample damage can result. It is therefore better to decouple the substrate bias using an inductively coupled rf plasma source. By using an inductively coupled plasma in conjunction with the full cusp magnetic field in the sample chamber, the plasma electrons can be confined to boost the plasma density and enhance its uniformity. In addition, the inductively coupled rf plasma source has the following advantages:

(1) relatively pure plasma due to electrodeless discharge;

(2) reasonably high plasma density;

(3) minimal contamination;

(4) small substrate damage;

(5) higher atomic ion ratio and multiply charged ion content (higher plasma chemical activity);

(6) suitable for active gases.

A $13.56 \mathrm{MHz} 2 \mathrm{~kW}$ rf plasma source is mounted onto the central part of the top flange. The discharge chamber is a Pyrex cylinder $20 \mathrm{~cm}$ in diameter and $30 \mathrm{~cm}$ in height. The rf power is fed into the discharge chamber through a watercooled inducting coil with seven turns to achieve optimal performance. A solenoid with 3920 turns surrounds the discharge chamber to sustain the rf discharge and enable the rf plasma to diffuse into the main vacuum chamber. Four different gases can be mixed and fed into the chamber through the rf coil. Two mass flow meters with ranges of $100 \mathrm{sccm}$ are employed to manipulate the gas flow. A multiple aperture electrode forms the extractor of the plasma source. When a positive potential is applied to the electrode, electrons can be extracted from the rf plasma. Conversely, ions can be extracted using a negative bias. When the multiaperture electrode is at ground potential, the rf plasma can diffuse into the main chamber. The rf plasma density can be readily altered by adjusting the rf input power and working gas pressure in the rf plasma source.

In order to assess the performance of the rf source, the plasma density is measured by a Langmuir probe as a function of the rf input power at three different working gas pressures (Fig. 3). At the same working gas pressure, the plasma density goes up when the rf input power is increased. At the same rf input power, the plasma density is greater at a working gas pressure of $1 \mathrm{~Pa}$, and lower at a working gas pressure of $0.5 \mathrm{~Pa}$. The plasma density as a function of the working gas pressure at various rf input power settings is shown in Fig. 4. The plasma density peaks at a pressure of 1-2 Pa. For the same working gas pressure, the plasma density goes up with increasing rf input power. When used simultaneously with our built-in antenna system (the $2 \mathrm{~kW}$, 13.56 $\mathrm{MHz}$ rf generator is capacitively coupled to the 4 


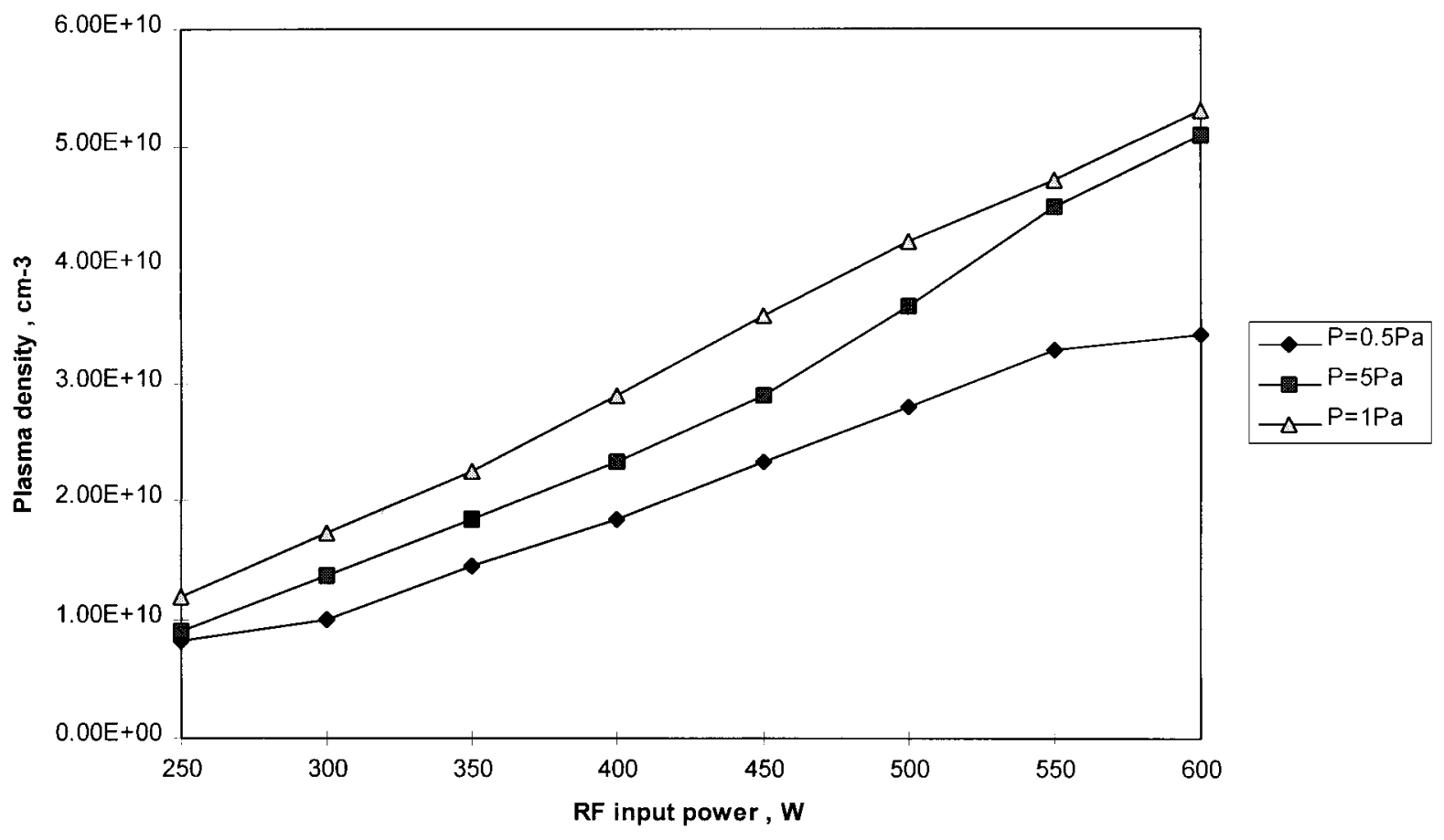

FIG. 3. Plasma density at various rf input powers.

$\mathrm{cm} \times 90 \mathrm{~cm}$ aluminum sheet antenna through special feedthroughs), the radial distribution of the plasma density is quite good (Fig. 5). Our results show that the coupling with a rf antenna exciting system can produce a fairly uniform plasma density both radially and axially. Based on its operating characteristics, the optimal working parameters are 400 $\mathrm{W}$ input power and $1 \mathrm{~Pa}$ pressure. Under these conditions, the plasma density is $2.87 \times 10^{10} \mathrm{~cm}^{3}$. The radial distribution uniformity of plasma density is better than $97 \%$ throughout a circular zone $60 \mathrm{~cm}$ in diameter.

\section{Multifilament modules: Hot filament discharge}

The advantages of hot filament modules are that they are economical as well as easy to operate, maintain, and replace. However, their life span tends to be short and the plasma can be contaminated by particles emitted from the filaments. Four sets of multifilament modules are inserted though the sidewall or the top flange of the main chamber for excitation of the plasma (Fig. 6). When the negatively biased filaments are heated, thermal electrons are emitted to ionize the gas-

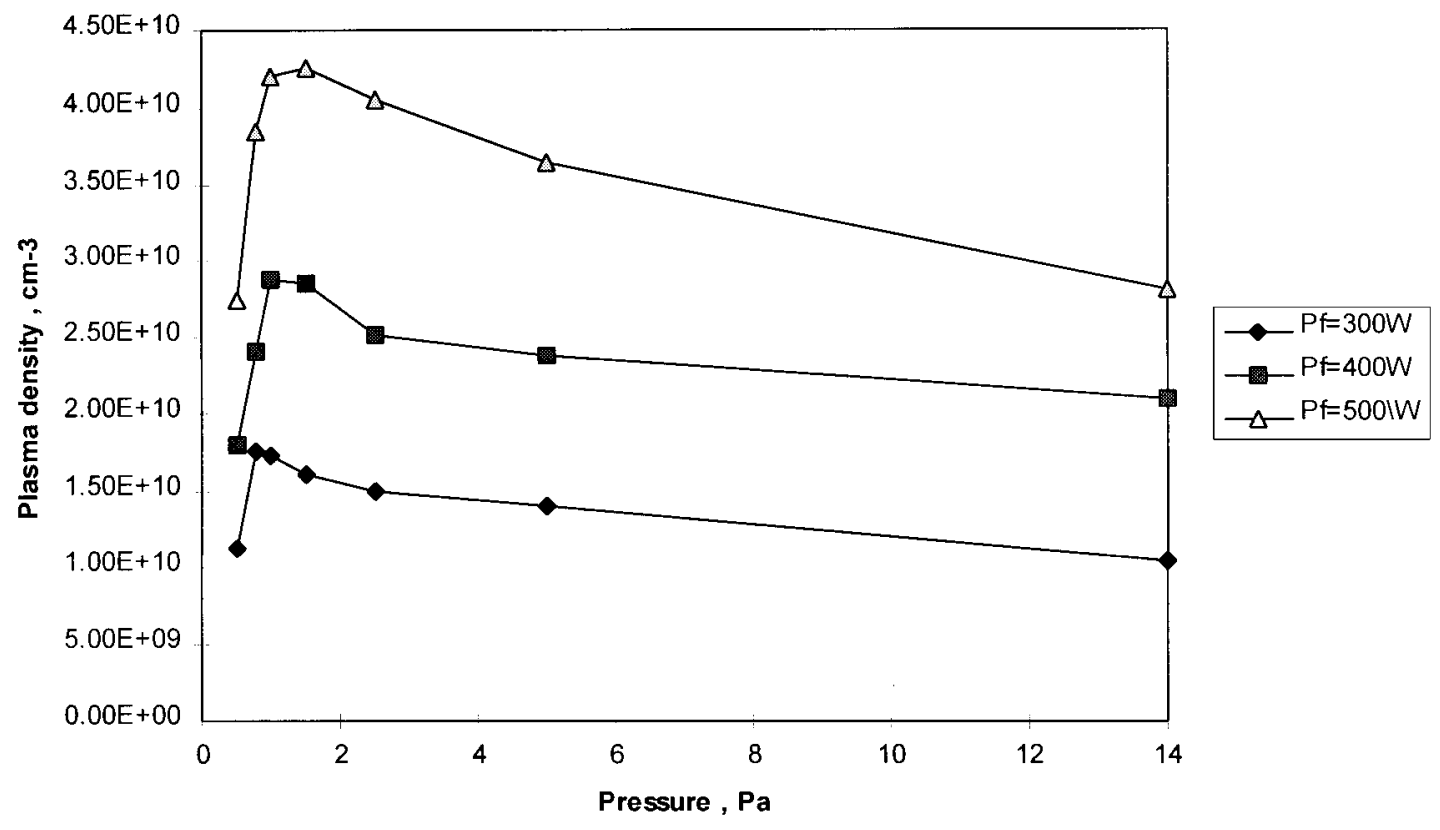

FIG. 4. Plasma density at various gas pressures. 


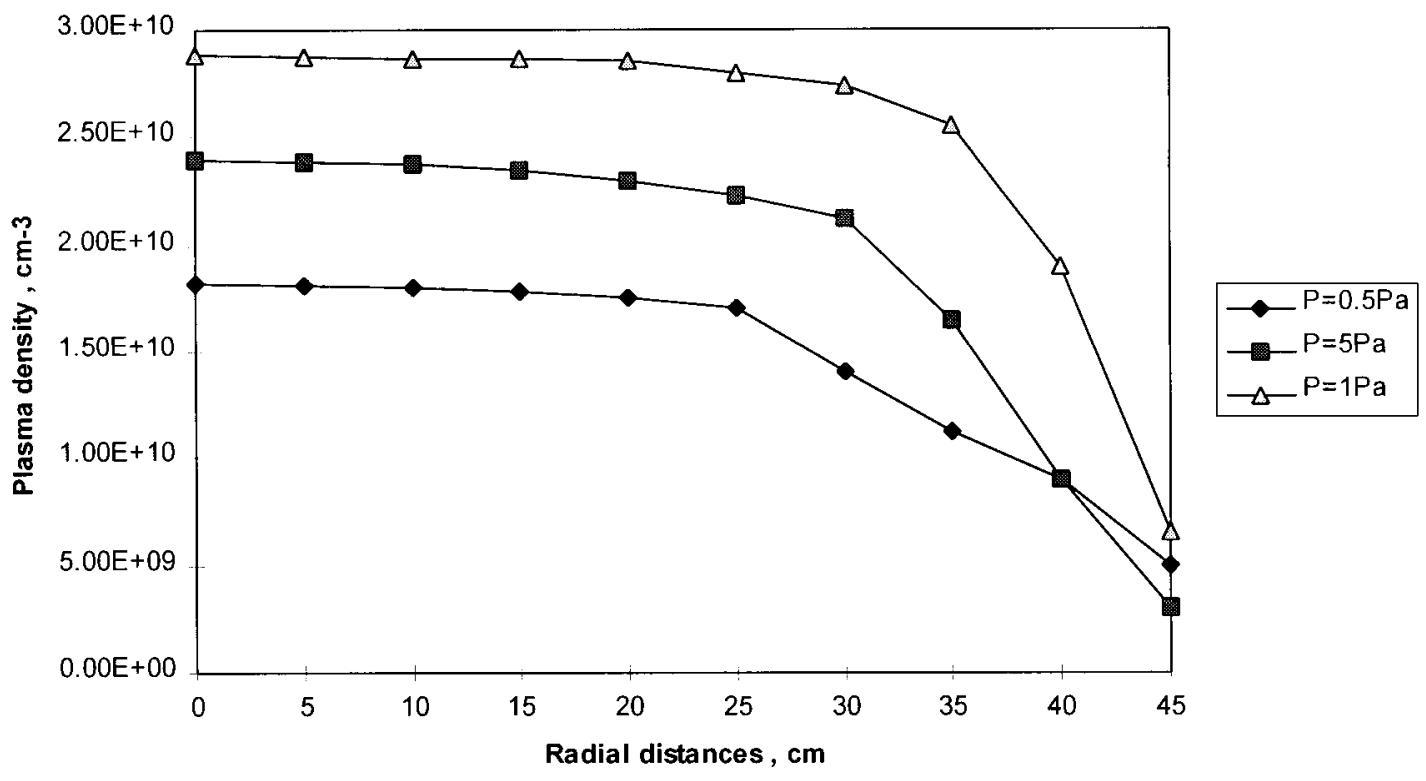

(a)

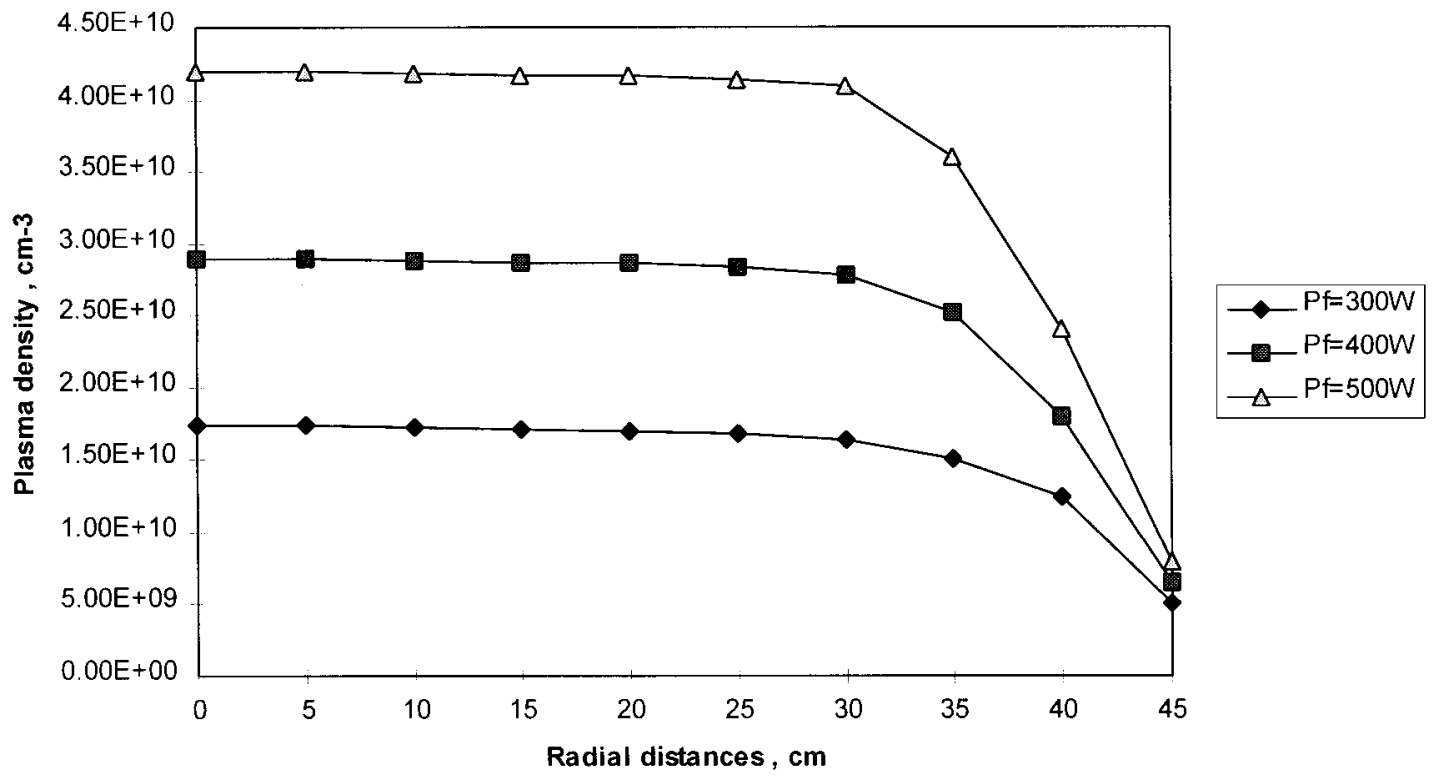

FIG. 5. Radial distribution of plasma density for (a) different gas pressures and (b) different rf input powers.

eous species in the plasma. In order to provide enough primary electrons, four sets of multifilament modules are symmetrically positioned in the chamber. The filament operating voltage is $0-40 \mathrm{~V}$ and the resulting filament current is $0-30$ A. Numerous $\mathrm{Th}-\mathrm{W}$ wire filaments are spot welded parallel on the multifilament module. The discharge bias which is adjustable from 0 to $150 \mathrm{~V}$ is applied between the filaments (cathode) and the chamber wall (ground) to yield a discharge current of 0-20 A. All the multifilament modules are in a magnetic field free region. The plasma density can be easily altered by varying the filament heating current, filament bias, and working gas pressure. The hot filaments are usually used for testing and equipment tuning. For applications requiring a clean ambient, the antenna system described in Sec. III B is used to ensure a uniform plasma distribution within the chamber.

\section{Solid ion sources}

Metal plasma sources based on vacuum arc discharge are capable of producing intense, fully ionized metal and carbon plasmas. ${ }^{17}$ Hence, metal plasma immersion (MPI) ion implantation can be performed when the substrate is negatively biased and low energy plasma deposition can be achieved when the bias pulse is off. This MPI technique opens up new research areas, such as atomic mixing, stress reduction, and formation of novel structures.

Four sets of MEVVA plasma sources ${ }^{18,19}$ are inserted 


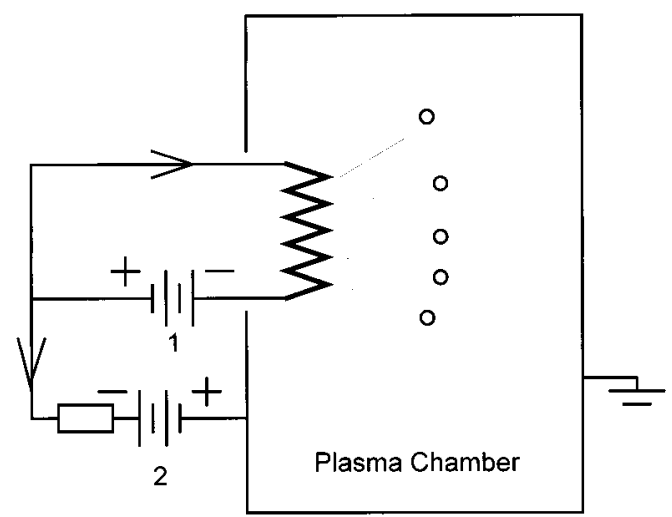

1.Filament Power Supply 2.Discharge Power Supply

FIG. 6. Hot electron filament module.

into the vacuum chamber as shown in Fig. 7, so that different metallic ions can be introduced into the plasma at the same time. If the same target is used for each of the sources, the introduction rate and consequently the metal ion density can be boosted. It is believed that our instrument is the first one with this multiple arc source design. Because macro and neutral particles can have deleterious effects on the film quality, particularly on adhesion between the film and substrate, they must be removed from the metal plasma using a special magnetic duct (filter). Diaphragms on the entrance and exit of the magnetic duct can further improve the filtering efficiency. The magnetic duct makes a $45^{\circ}$ angle. Its axial magnetic field can be varied from 0 to $35 \mathrm{mT}$ and the exit aperture is $80 \mathrm{~mm}$ in diameter. As mentioned earlier, when the substrate is at ground potential, deposition occurs and, if a negative bias is applied to the substrate, ion implantation takes place. Hence, either deposition or implantation can be carried out by varying the ratios between the pulse width of the metal arc and the width of the negative bias pulse. The operating parameters of the four MEVVA plasma sources are pulse duration: $\sim 200 \mu$ s, triggering voltage: $10 \mathrm{kV}$, arc voltage: $100 \mathrm{~V}$.

The four sets of arc pulses can be synchronized and different metal plasmas can be obtained by replacing the cathode materials. The metal plasma density at the exit of the magnetic duct is $10^{9}-10^{12} \mathrm{~cm}^{-3}$.

To complement the MEVVA sources, there is also a sputtering electrode system in our instrument for introducing solid ions into the plasma. ${ }^{20}$ It is composed of a sputtering electrode, a shutter, a $2 \mathrm{~kW}$ dc power supply, and a 13.56 $\mathrm{MHz} 2 \mathrm{~kW}$ rf generator. In the dc sputtering mode, the cathode which is made of the sputtering materials is biased negatively while the substrate (anode) is usually grounded. Deposition can be followed by implantation by applying a large negative potential to the substrate. If the sputtering target, substrate, or deposited film is an insulator, a rf glow discharge is needed to overcome charging effects. The electron oscillation in the rf glow discharge is so intense that electrons gain enough energy to ionize the species in the plasma. Consequently the breakdown voltage and dependence of the glow discharge on secondary electrons are reduced and the rf

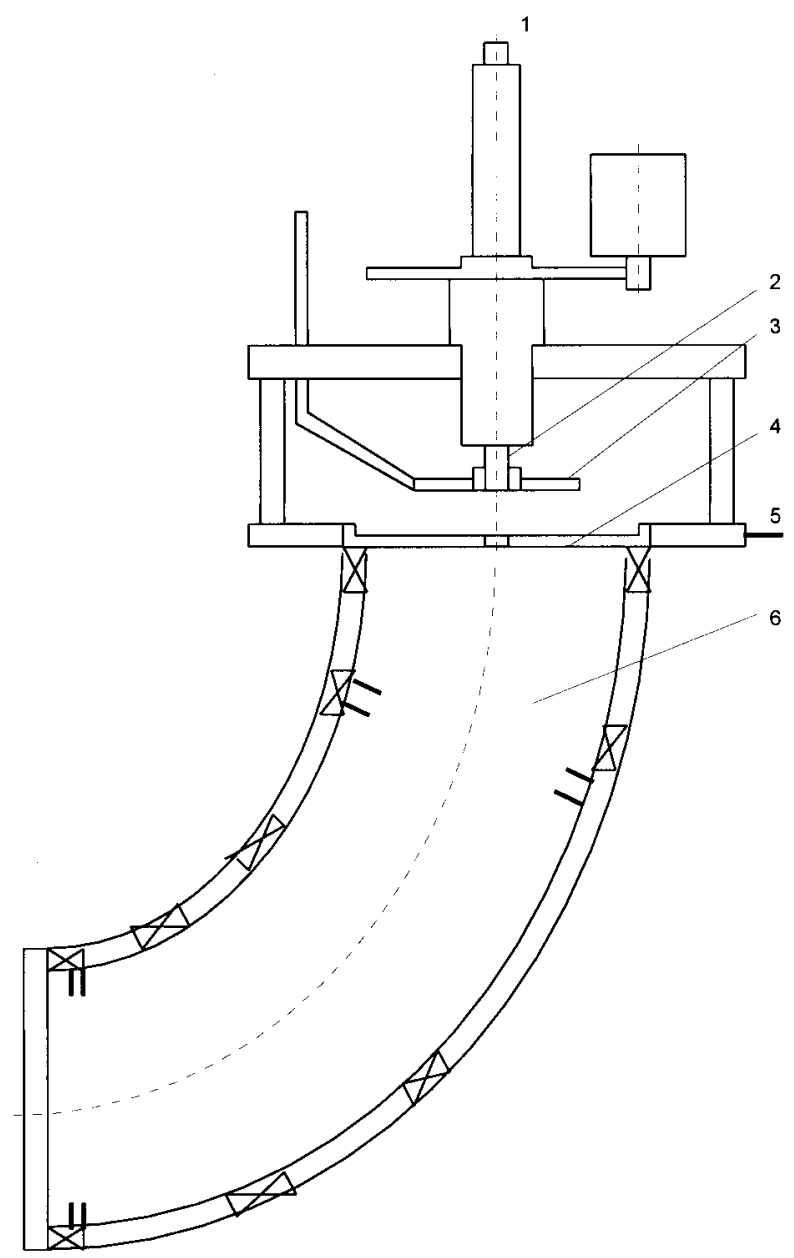

FIG. 7. MEVVA plasma source (1) Water cooling tube; (2) cathode; (3) triggering electrode; (4) anode; (5) water cooling tube; (6) magnetic duct.

power can be coupled into any type of impedance. Sample charging can also be alleviated by electrons attracted from the plasma; hence insulators can be used as electrodes. A rf capacitively coupled glow discharge can be initiated and maintained at a lower pressure than its dc counterpart. It operates at $5-30 \mathrm{MHz}$ and its output voltage is less than the bias voltage of a de glow discharge. In the City University system, a commercial $13.56 \mathrm{MHz}$ rf generator is used. Important operating parameters of the rf generator, including amplitude, repetition rate, and voltage pulse duration, can be independently adjusted to achieve uniform deposition without significant heating of the substrate. The ion concentration gradient and surface temperature can be individually regulated as well.

It should be emphasized the ability to perform ion implantation and sputter deposition in the same chamber is important because deleterious effects caused by exposure to air can be eliminated. Our instrument therefore excels in treatment processes that require multiple implantation and thin film deposition during which samples cannot be exposed to air in between steps. ${ }^{21}$

\section{E. Pumping system}

As seen in Fig. 8, the pumping system is composed of a ZBS-250 gate valve, a F-250 turbomolecular pump, a 150 


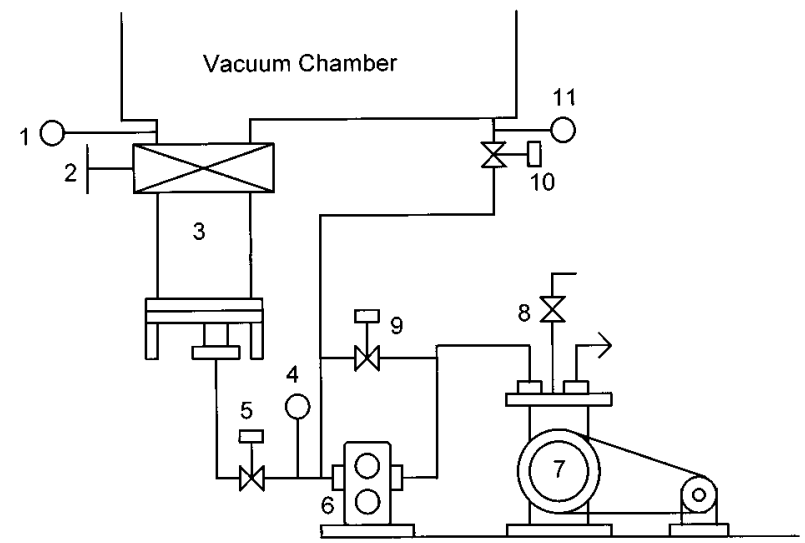

FIG. 8. City University's PIII vacuum pumping system. (1) Ionization gauge; (2) ZBS-250 gate valve; (3) turbomolecular pump; (4) TC1; (5) GDC-J65; (6) Roots vacuum pump; (7) 2X-30 mechanical pump; (8) DDCJQ65; (9) GDC-J65; (10) GDC-J65; (11) TC2.

Roots vacuum pump, and a $2 \mathrm{X}-30$ mechanical pump. A turbomolecular pump is chosen in lieu of a diffusion pump and cryogenic pump because of oil contamination (by the diffusion pump) and safety issues (condensation and evolution of hazardous gases from the cryogenic head). The effective pumping speed of the main pump is approximately $940 \ell / \mathrm{s}$. There also a port where a high capacity cryogenic pump can be installed.

When a high working pressure is required, as for example, when a glow discharge is activated, the 150 Roots vacuum pump is kicked in. When ion implantation is performed, the Roots pump is turned off and electromagnetic valve No. 9 [Fig. 8] is opened. The thermocouple gauges (TC1 and TC2) and the ZDO-3 digital thermocouple vacuum meter provide instantaneous and accurate monitoring of the total pressure from $10^{-3}$ to 2.0 Torr. For lower pressure $\left(9 \times 10^{-4}-10^{-10}\right.$ Torr), the ionization gauze (IG) and ZDR- 8 intelligent vacuum meter are used. The ZBS-250 gate valve isolates the chamber from the pumps. When the gate valve is opened, the chamber is connected to the pumping system and can be evacuated. The chamber is vented by closing the gate valve before purging. The gate valve can also be partially opened to control the pressure inside the chamber.

\section{F. High voltage pulse power supply}

Ion implantation is usually performed at a high implantation voltage, low implantation temperature, and low working gas pressure whereas coating and sputter deposition are carried out at a low implantation voltage, high temperature, and high working gas pressure. For semiconductor applications such as fabrication of silicon-on-insulator, the typical conditions call for a medium to high implantation voltage $(>50 \mathrm{kV})$. Hence the equipment is designed to operate from a few $\mathrm{kV}$ to a maximum sample voltage of $100 \mathrm{kV}$. The 100 $\mathrm{kV}$ upper ceiling is due to the fact that safe and arcing-free routine operation will be difficult if the sample bias is more than $100 \mathrm{kV}$.

The high voltage pulse power supply ${ }^{22}$ is comprised of a dc high voltage power supply, a main charging capacitor, a pulse modulator, and a high voltage pulse transformer (Fig.

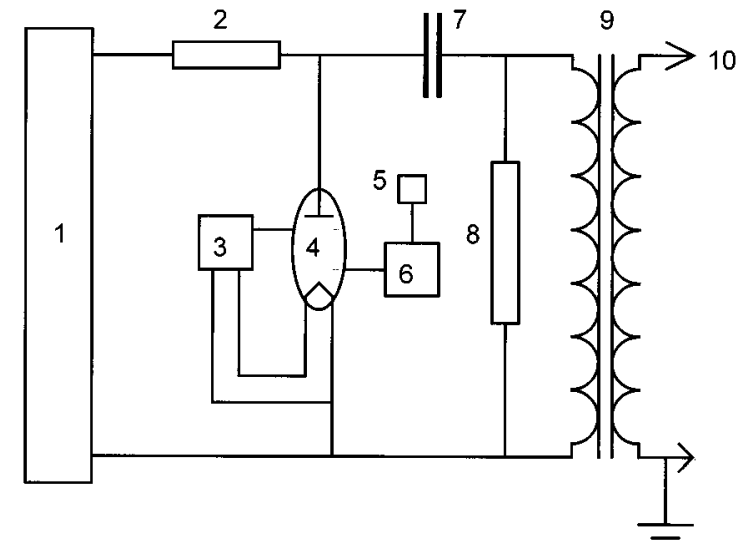

FIG. 9. Simplified diagram of the high voltage pulse power supply. (1) dc high voltage power supply; (2) overcurrent protection; (3) auxiliary power supply of tetrode; (4) tetrode; (5) pulse signal generator; (6) pulse power amplifier; (7) main capacitor; (8) charging and protector circuit; (9) high voltage pulse transformer; (10) workpieces.

9). The divider, main capacitor, tetrode, high voltage transformer, and other components are submerged in recirculating cooling oil. The main capacitor is normally charged by the dc high voltage power supply during operation. The modulator consists of a pulse generator, pulse grid controller, and high voltage tetrode tube. The pulse generator supplies a small pulse (up to $10 \mathrm{~V}$ ) to the grid controller which in turn furnishes pulses of several hundred volts to the grid of the tetrode tube. The tetrode switches the main charging capacitor on and off. The high voltage pulse rises to the desired level before it is applied to the substrate. When the tetrode is grounded, one side of the main capacitor is also grounded and it is discharged via the high voltage pulse transformer.

The pulse on the substrate can be monitored by a simple voltage divider in which there are $1001 \mathrm{M} \Omega$ resistors in series. The total potential difference across the divider is uniformly distributed among each resistor and the uniform electric field offers better reliability. There is a $0.1 \mathrm{k} \Omega$ variable resistor and a combination of resistors and capacitors to tune the shape of the voltage pulse. The output trace from the divider is calibrated by a Tektronics high voltage probe and a digital oscilloscope. During normal operation, the trace is continuously monitored on the oscilloscope and the substrate potential is measured. A current transformer on the high voltage power supply is employed to monitor the wave form and measure the total implantation current.

The operating parameters of the high voltage pulse power supply are pulse rise time: $\sim 100 \mathrm{kV} / \mu \mathrm{s}$, pulse duration: $10-60 \mu \mathrm{s}$, pulse repetition rate: $10-5000 \mathrm{~Hz}$, pulse amplitude: 5-100 kV, maximum pulse current: $10 \mathrm{~A}$, maximum average output current: $80 \mathrm{~mA}$.

Because the total implantation time, $T(s)$, is directly affected by the operating parameters of the high voltage pulse power supply, they must be controlled and monitored accurately. The implantation time can be expressed by the following relationship:

$$
T=\frac{\left(1.6 \times 10^{-19} D A\right)(1+\gamma)}{t_{p} f N\left(I_{m}-I_{d}\right)},
$$


where $D$ is the implantation dose in atoms $/ \mathrm{cm}^{2}, A$ is the total implantation area in $\mathrm{cm}^{2}, t_{p}$ is the high voltage pulse width in $\mathrm{s}, f$ is the pulse repetition frequency, $\gamma$ is the secondary electron coefficient of the substrate, $N$ is the average number of atoms produced per implanted ion in the implanted layer, $I_{m}$ is the average implantation current measured by the oscilloscope, and $I_{d}$ stands for the average current measured by the oscilloscope in the absence of the plasma.

\section{G. High voltage target stage and oil cooling system}

The high voltage target stage is comprised of a top copper flange, a ceramic insulator, a high voltage feedthrough, a bottom flange, and an oil barrel. The ceramic insulator is thick enough that electrical isolation between the top copper flange and the bottom of the chamber can be attained at a $100 \mathrm{kV}$ applied bias. The stage measures $250 \mathrm{~mm}$ in height and $76 \mathrm{~mm}$ in inside diameter. A negatively bias pulsed voltage is applied to the top copper flange through the high voltage feedthrough which is a copper tube $20 \mathrm{~mm}$ in diameter. In order to remove excessive heat produced by the substrate during ion implantation or sputter deposition, the top copper flange is cooled with recycling oil from an oil cooling system that is composed of an oil barrel, an oil pump, and a valve relay. A $100 \mathrm{kV}$ high voltage shielded cable can be directly inserted into the high voltage feedthrough.

\section{H. Protection, control, and safety features}

The City University system is designed to ensure safe, precise, and efficient operation. The protection, control, and operation electronics are housed in five racks. The filament power supply, arc discharge power supply, and Langmuir probe bias power supply are in the plasma control rack, as well as the manual control, protection, operation, and measurement circuits necessary to plasma production. The corresponding knobs and displays are on the front panel of the cabinet.

The ZDR-8 type intelligent vacuum meter, ZDO-3 type digital thermocouple vacuum meter, flow display power supply, turbomolecular pump power supply, and the corresponding circuits are accommodated on the vacuum rack. The MEVVA rack houses the high voltage trigger power supply, arc discharge power supply, and magnetic field power supply of the MEVVA sources. The dc high voltage power supply, rigid modulation circuits, etc. can be found in the two high voltage pulse power supply cabinets. All five racks are interconnected for safety.

For personal and for equipment safety, certain logics are implemented into the City University equipment. The sample high voltage cannot be triggered on or automatically turned off when the chamber door is not closed, when there is a sudden pressure surge, in case of high voltage arcing or breakdown, or when the water or oil cooling system fails.

In addition, certain additional precautions are included, before targets are removed from the high voltage sample stage, the grounded safety bar must be in contact of the stage. Self-locking devices ensure that wrong operating steps cannot be executed. Current or voltage protection kicks in automatically when such failure occurs. When the pressure

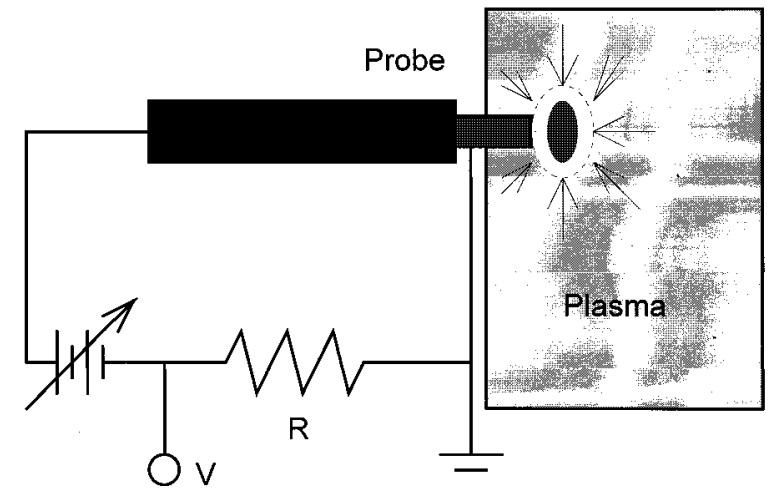

FIG. 10. Langmuir probe measurement system.

exceeds $10^{-3}$ Torr, the ion gauges go off automatically. When the chamber is accidentally vented, the gate valve closes immediately.

\section{Plasma diagnostics}

For accurate control of the processes, important parameters must be monitored. These include plasma density and uniformity, electron temperature, ion temperature; plasma sheath edge propagation, ion energy distribution on the plasma sheath, ion species, secondary electron emission coefficient of the targets, charge state of the ions, and plasma oscillation. In our equipment, the Langmuir probes are inserted into the chamber through special openings along the radial and axial directions. The probes are made of tantalum because of its low sputtering coefficient and good conduction and are connected to the power supply via a plated gold wire. The Langmuir probe system consists of the probe, drive system, bias power supply, measurement circuit, $x-y$ recorder, and oscilloscope.

The current-voltage $(I-V)$ characteristics of Langmuir probes are very useful in determining plasma parameters such as plasma density, plasma potential, and electron/ion temperature. Langmuir probes function well at plasma densities of $10^{0}-10^{13} \mathrm{~cm}^{-3}$, temperature from 0.1 to hundreds of $\mathrm{eV}$, plasma potentials from $0.1 \mathrm{~V}$ to several $\mathrm{kV}$, and at a neutral pressure between $10^{-6}$ and over 1 Torr. Figure 10 shows the Langmuir probe measurement system, and the $I-V$ characteristics are displayed in Fig. 11. The mathematical relationship is

$$
\begin{aligned}
& I_{e}\left(V_{B}\right)=I_{e}^{*} \exp \frac{-e\left(V_{p}-V_{b}\right)}{T_{e}}, \quad V_{B} \leqslant V_{P}, \\
& I_{e}=I_{e}^{*}, \quad V_{B} \geqslant V_{P}, \\
& I_{e}^{*}=A n_{e} e \sqrt{T_{e} / 2 \pi m_{e}}, \\
& I_{i}^{*}=0.6 A n_{i} e \sqrt{T_{e} / m_{i}},
\end{aligned}
$$

where $V_{B}$ is the bias voltage applied to the probe, $V_{P}$ is the plasma potential, $I_{e}^{*}$ is the electron saturation current, $I_{i}^{*}$ is ion saturation current, $n_{e}$ is the electron density, $n_{i}$ is the ion density, $n_{e}=n_{i}=n$ plasma density, and $T_{e}$ is the electron temperature. According to the $(I-V)$ characteristics of the probe, the plasma density, electron temperature, and plasma potential can be calculated. As demonstrated in Fig. 11, the 


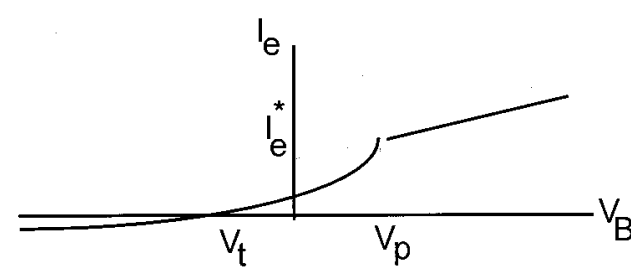

FIG. 11. $I-V$ characteristics of the Langmuir probe.

plasma potential $V_{P}$ can be approximated by the intersection of two straight lines, one parallel to the slanted part and the other parallel to the curve above the kink. Other plasma diagnostic tools include a Faraday cup, a current probe, a microwave detector, a sampling ion source-mass filter, laser induced fluorescence, and an Omegatron mass spectrometer or quadrupole mass filter.

\section{ACKNOWLEDGMENTS}

The design and construction of the PIII equipment was financially sponsored by the City University of Hong Kong. Hong Kong University of Science and Technology, and the Hong Kong Research Grants Council Central Allocation (Contract No. 8730005).

${ }^{1}$ J. K. Hirvonen, C. A. Carosella, R. A. Kant, and I. Singer, Thin Solid Films 63, 5 (1979).

${ }^{2}$ S. T. Pictraux and P. S. Thomas, Phys. Today 37, 38 (1984).

${ }^{3}$ R. Hutchings and W. C. Oliver, Wear 92, 143 (1983).
${ }^{4}$ G. Dearnaley, Nucl. Instrum. Methods 87, 158 (1985).

${ }^{5}$ A. J. Armini and S. N. Bunker, Mater. Sci. Eng. A 115, 67 (1989).

${ }^{6}$ J. R. Conrad, J. L. Radtke, R. A. Dodd, F. J. Worzale, and N. C. Tran, J. Appl. Phys. 62, 4591 (1987).

${ }^{7}$ J. R. Conrad, J. Appl. Phys. 62, 777 (1987).

${ }^{8}$ J. R. Conrad, U. S. Patent No. 476,494 filed 16 August 1988.

${ }^{9}$ S. H. Han, G. L. Kulcinski, and J. R. Conrad, Nucl. Instrum. Methods Phys. Res. B 45, 701 (1990).

${ }^{10}$ F. J. Worzala, R. A. Dodd, and J. R. Conrad, 7th International Conference Ion and Plasma-Assisted Techniques, Geneva, Switzerland, 31 May-2 June, 1989.

${ }^{11}$ X. Qiu, J. R. Conrad, R. A. Dodd, and F. J. Worzala, Proceedings of the 1988 AIME Fall Meeting, September 1988.

${ }^{12}$ J. R. Conrad, R. A. Dodd, F. J. Worzala, and X. Qiu, Surf. Coat. Technol. 36, 927 (1988).

${ }^{13}$ B. Y. Tang, R. P. Fetherston, M. Shamin, R. A. Breun, A. Chen, and J. R. Conrad, J. Appl. Phys. 73, 4176 (1993).

${ }^{14}$ P. K. Chu, Y. C. Cheng, and P. K. Ko (unpublished proposal to RGC Hong Kong, 1994).

${ }^{15}$ L. M. Yang, Mechanical Design Handbook of Mechanical Parts (China National Defense Industry, 1986).

${ }^{16}$ F. Mahboubi, M. Samandi, and D. Dunne, Surf. Coat. Technol. 85, 44 (1996).

${ }^{17}$ G. Brown, A. Anders, S. Anders, M. R. Dickinson, and R. A. MacGill, J. Vac. Sci. Technol. B 12, 823 (1994).

${ }^{18}$ S. Anders, A. Anders, and I. G. Brown, 5th International Conference on Ion Sources, Beijing, China, 31 August-4 September 1993.

${ }^{19}$ A. A. Elmoursi, G. W. Malaczynski, and A. H. Hamdi, Nucl. Instrum. Methods Phys. Res. B 62, 293 (1991).

${ }^{20}$ P. Zhu and R. W. Boswell, J. Appl. Phys. 68, 1981 (1990).

${ }^{21}$ S. Y. Wang, X. C. Zeng, Y. Gao, A. G. Liu, X. F. Wang, D. Z. Xing, X. B. Tian, B. Y. Tang, and P. K. Chu, Proceedings of IBMM '96, Albuquerque, NM, 1996, p. 314

${ }^{22}$ A. A. Elmoursi, G. W. Malaczynski, and A. H. Hamdi, Nucl. Instrum. Methods Phys. Res. B 62, 293 (1991). 